\title{
Thermal Reaction Characterization of an Epoxy Molding Compound
}

\author{
Man Geng Lu, Hyeong-Ki CHOI, ${ }^{*}$ Hong-Ki LEE, ${ }^{* *}$ Mi-Ja SHIM, ${ }^{* * *}$ and Sang Wook KIM ${ }^{* * * *, ~}{ }^{\dagger}$ \\ Fine Polymer Co. Ltd., 629-7 Seongkok-Dong, Ansan 425-110, Korea, \\ ${ }^{*}$ Division of Biotechnology and Environmentel Eng., NITQ, Kwacheu 427-010, Korea, \\ ${ }^{* *}$ Department of Chem. Eng., WooSuk University, Wanju 565-800, Korea, \\ ${ }^{* * *}$ Department of Life Sci., The University of Seoul, Seoul 130-743, Korea, \\ ${ }^{* * * *}$ Department of Chem. Eng., The University of Seoul, Seoul 130-743, Korea
}

(Received June 12, 2000; Accepted December 15, 2000)

\begin{abstract}
A novel epoxy molding compound for electronic industry application was used to conduct thermal analysis characterization using differential scanning calorimetry (DSC) and thermogravimetric analyzer (TGA). The reaction kinetics of cure and thermal degradation have been analyzed based on a proposed iso-conversional model. The kinetic results showed that the cure and decomposition process are complex, and the values of activation energy are dependent on the degree of conversion.

KEY WORDS Epoxy Molding Compound / Iso-Conversional Model / Cure Kinetics / Thermal Degradation /
\end{abstract}

Electrical and electronic devices have been encapsulated in a variety of resinous materials, including epoxy, silicone and phenolic materials. Epoxy molding compounds for the encapsulation of electronic components are considered a specialty market by the epoxy manufactures. The worldwide consumption of electronic grade molding compounds is approximately 100000000 metric tons (1997) and growing. The amount of epoxy resin used in these compounds is approximately $10 \%$ of that figure. The electronic grade epoxy resins must have a higher degree of purity than the commodity epoxy resins. The halogen content of these resins is usually less than $800 \mathrm{ppm}$ and are much more difficult to manufacture than the standard resins. ${ }^{1}$

The useful properties of epoxy resins appear only after curing. The curing step transforms the epoxy from a lowmolecular-weight material to a highly crosslinked space network. The properties of the cured resin depend on either the type of epoxide and the curing system used or the extent of cure. Recently, epoxy molding compounds have been widely used in the electronic industry and encapsulation of integrated circuits by means of transfer molding. ${ }^{2}$ Only limited information, however, may be found in the literature concerning the cure and degradation characterization of these molding compounds. Therefore, kinetic characterization of thermoset systems is necessary for a better understanding of structureproperty relationships. ${ }^{3}$

The attractive feature of isothermal experiments is that the rate constants at each temperature are better defined and the constants obtained at different temperatures would permit the determination of the activation energy associated with the thermal degradation. ${ }^{4,5}$ However, the nature of the reactions and the final products may differ at different temperatures, and the kinetic parameters thus obtained are not without ambiguity. ${ }^{6}$

Dynamic experiments, conducted at a specified heating rate using DSC, will yield conversion-timetemperature data that are comprehensive enough to per- mit direct evaluations of the kinetic parameters. A single dynamic run gives as much information as do several isothermal runs. Furthermore, dynamic measurements can provide kinetic information over a larger temperature range and there is no preheating problems as is the case with isothermal experiments in which the sample must be first heated to the isothermal hold temperature during which thermal reactions may take place. It is valuable as a precursor to isothermal studies and is often the only means to analyze the cure kinetics of systems with multiple exotherms. ${ }^{7}$ One shortcoming of this method based on the mechanistic model is the requirement of an assumed reaction order.

Comparing with the isothermal method, the case of dynamic cure is theoretically more difficult for the phenomenological kinetic model due to the complex temperature dependence of rate constant and the peculiar feature of cure, although it is a case which more closely simulates the fabrication process. ${ }^{8}$

In this paper, an isoconversional analytical model was proposed, where $f(\alpha)$ has the same form regardless of the temperature for the same degree of conversion $\alpha$. The kinetic parameters of cure and degradation of epoxy molding compounds, and the dependence of activation energy on conversion were described on the basis of DSC and TGA measurements.

\section{Theoretical Analysis}

Different methodologies can be used to evaluate kinetic parameters and are available on the cure and degradation behavior of epoxy resins. In general, kinetic expressions may be phenomenological or mechanistic. All kinetic studies can start with the basic equation that relates the rate of conversion at constant temperature to some function of the concentration of reactants:

$$
\frac{\mathrm{d} \alpha}{\mathrm{d} t}=k f(\alpha)
$$

or in the integrated form:

\footnotetext{
${ }^{\dagger}$ To whom correspondence should be addressed (Phone: +82-2-2210-2447, Fax: +82-2-2210-2310, E-mail: swkim@uoscc.uos.ac.kr).
} 


$$
g(\alpha)=\int \frac{d \alpha}{f(\alpha)}=\int k \mathrm{~d} t
$$

where $\frac{\mathrm{d} \alpha}{\mathrm{d} t}$ is the rate of reaction; $\alpha$ is the fractional conversion at any time $t ; k$, the Arrhenius rate constant, and $f(\alpha)$, a function form of $\alpha$ that depends on the reaction mechanism.

In our previous work, the kinetic parameters from various published models have been compared and used to elucidate the thermal reaction behavior for several epoxy resin systems. ${ }^{9}$ It has also been observed that for the kinetic analysis involved multiple constant heating rates, Osawa and Kissinger methods are superior to other methods. ${ }^{10}$

In the case of dynamic curing, when the temperature varies with time with a constant heating rate, $\beta=\mathrm{d} T / \mathrm{d} t$, eq 2 is represented as follows:

$$
\int \frac{d \alpha}{f(\alpha)}=\int \frac{A}{\beta} \exp \left(-\frac{E_{\mathrm{a}}}{R T}\right) \mathrm{d} T
$$

where $A$ is the pre-exponential factor, $E_{\mathrm{a}}$ is the activation energy.

Integration of eq 4 proceeds as follows:

$$
\begin{aligned}
& g(\alpha)=\int_{0}^{\alpha} \frac{\mathrm{d} \alpha}{f(\alpha)}=Z \int_{0}^{t} e^{-E / R T} \mathrm{~d} t=\frac{Z}{\beta} \int_{T_{0}}^{T} e^{-E_{\mathrm{a}} / R T} \mathrm{~d} T \\
& \approx \frac{Z}{\beta} \int_{0}^{T} e^{-E_{\mathrm{a}} / R T} \mathrm{~d} T \approx \frac{A E_{\mathrm{a}}}{\beta R} p\left(E_{\mathrm{a}} / R T\right)
\end{aligned}
$$

where $T_{0}$ is the initial temperature in the DSC analysis and $T$ is the final temperature, and

$$
p(x)=\int_{\infty}^{x} \frac{e^{-x}}{x^{2}} \mathrm{~d} x
$$

where $x$ is a polynomial $v s$. $\left(E_{\mathrm{a}} / R T\right)$.

For the kinetic study, many authors consider that, during the curing and degradation process, the reaction mechanism does not change and $f(\alpha)$ in eq 1 has the same form. In fact, the reaction process of thermosets is complex and, in many cases, it is not correct to consider that the reaction mechanism remains constant during the whole process, or that there is a single activation energy.

Herein, we consider that $f(\alpha)$ has the same form regardless of the temperature for the same degree of conversion $\alpha$. Thus, eq 3 can be integrated using the methods independently derived by Osawa ${ }^{11}$ and Flynn and Wall, ${ }^{12}$ and the activation energy $E$ can been given by plotting $\beta_{i}$ vs. $T_{\alpha, i}{ }^{-1}$ (here $i$ is ordinal numbers of DSC runs performed at different heating rates, $\beta_{i}$ ). Generally the $E$ values found by these methods require further correction which can be avoided when using the plot $\ln \left(\beta_{i} /\right.$ $\left.T_{\alpha, i}{ }^{2}\right)$ vs. $T_{\alpha, i}{ }^{-1.13,14}$

\section{EXPERIMENTAL}

\section{Materials}

The resin used in this study is a commercial mineral filled DGEBA-based epoxy compound (MG 6-0330, The Dexter Corporation, USA), which contains acid anhydride hardeners, about $72 \%$ of fused silica and $4 \%$ of carbon black, and other additives, according to the supplier. The samples for analysis of thermal degradation were

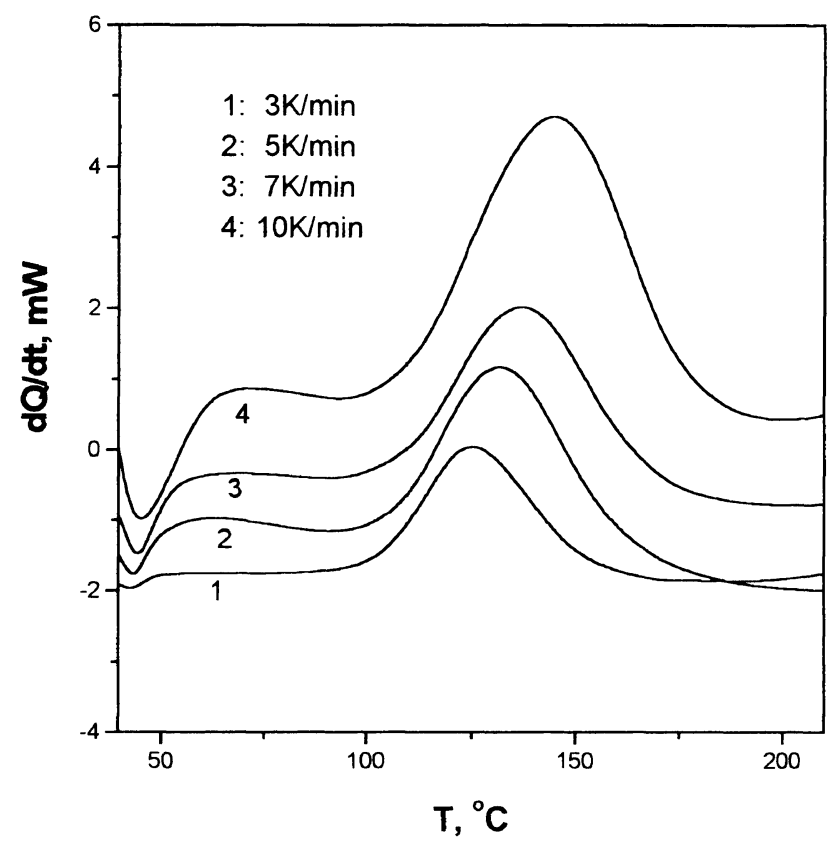

Figure 1. Dynamic scans of epoxy molding compounds at different heating rates.

processed by transfer molding at $135-177^{\circ} \mathrm{C}$ and postcured at $150^{\circ} \mathrm{C}$ for $2 \mathrm{~h}$.

\section{Dynamic Scans}

Dynamic curing were performed in a Solomat DSC 4000 at different heating rates from 3 to $20 \mathrm{~K} \mathrm{~min}^{-1}$ with $10-15 \mathrm{mg}$ of samples. A Instrumental Specialists Incorporated TGA-1000 Thermogravimetric Analyzer is used to obtain weight loss data for cured samples. Constant heating rates of $5,10,20$, and $50 \mathrm{~K} \mathrm{~min}^{-1}$ are used. The sample weights are in the range of $20-50 \mathrm{mg}$ in all cases and purged with nitrogen gas at a flow rate of $50 \mathrm{~mL} \mathrm{~min}^{-1}$. More details about the calibration procedure and thermal conditions can be found elsewhere. ${ }^{15}$

\section{RESULTS AND DISCUSSION}

\section{Curing Reaction}

The dynamic DSC thermograms at different heating rates are shown in Figure 1. It is seen that the cure exotherms are shifted to higher temperatures with increasing the heating rates. Although the DSC thermograms can shift by changing the amount of sample, we found that the curves have not been significantly affected in the limited weight range $(10-15 \mathrm{mg})$ as conducted in this experiments. The area under the exotherm is related to the curing heat, which is used to estimate the extent of cure. To perform iso-conversional analysis, the DSC scans were transformed into the form $\alpha_{i} v s . T_{i}$ for each $i$-th heating rate as shown in Figure 2 . It is clear that, for a given conversion, the larger the heating rate, the higher the temperature required.

The plots of $-\ln \left(\beta_{i} / T_{\alpha, i}{ }^{2}\right)$ vs. $T_{\alpha, i}{ }^{-1}$ are given in Figure 3 , resulting in the very good linear relations for the whole curing process. These results confirm that the basic idea of iso-conversional analysis is appropriate to describe the dynamic cure for the observed system. The values of activation energy, $E_{\mathrm{a}}$, for curing reaction, at 


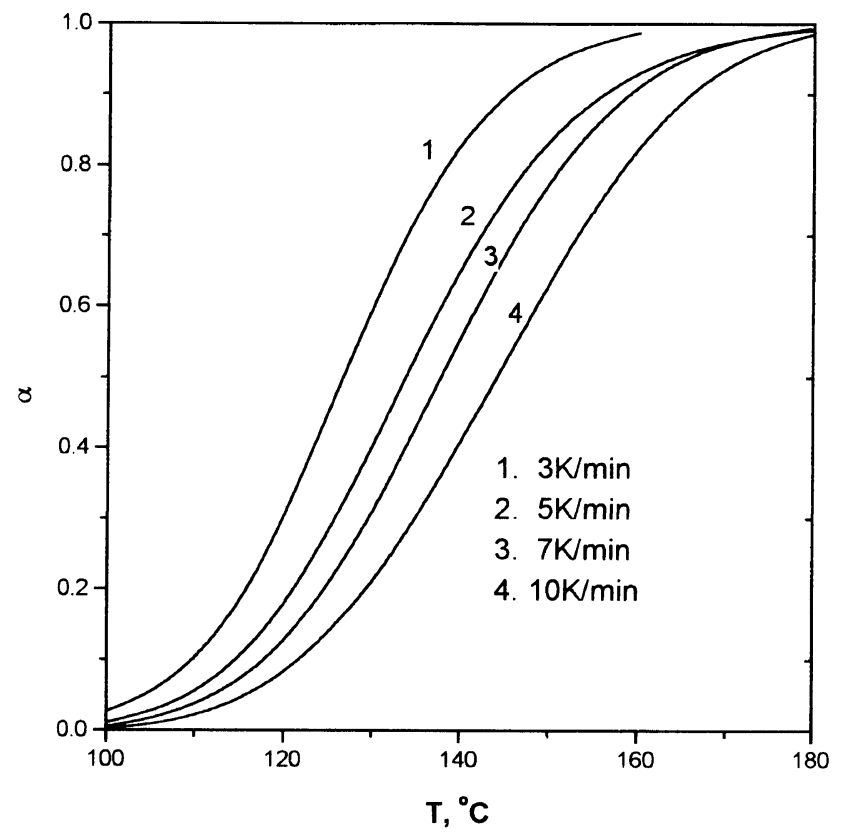

Figure 2. Degree of conversion vs. curing temperature at different heating rates.

Table I. Kinetic parameters analyzed by iso-conversional model

\begin{tabular}{|c|c|c|}
\hline Conversion & $\frac{\text { Activation energy }}{E_{a} / \mathrm{kJ} \mathrm{mol}}$ & $\frac{\text { Pre-exponential factor }}{A^{\mathrm{a} / \mathrm{min}^{-1}}}$ \\
\hline 0.1 & 121.8 & $8.73 \times 10^{12}$ \\
\hline 0.2 & 111.3 & $1.82 \times 10^{11}$ \\
\hline 0.3 & 101.1 & $5.48 \times 10^{9}$ \\
\hline 0.4 & 96.9 & $1.12 \times 10^{9}$ \\
\hline 0.5 & 87.6 & $5.33 \times 10^{8}$ \\
\hline 0.6 & 85.5 & $2.20 \times 10^{8}$ \\
\hline 0.7 & 82.9 & $7.76 \times 10^{6}$ \\
\hline 0.8 & 82.1 & $4.39 \times 10^{6}$ \\
\hline 0.9 & 81.3 & $2.12 \times 10^{8}$ \\
\hline
\end{tabular}

${ }^{\mathrm{a}} A=\beta E_{\mathrm{a}, \mathrm{p}} \exp \left(E_{\mathrm{a}, \alpha} / R T_{\alpha}\right) / R T_{\alpha}^{2}$

different conversions can be calculated from the slopes of the straight lines and have been listed in Table I. The dependence of $E_{\mathrm{a}}$ on $\alpha$ for the epoxy curing was given in Figure 4. The shape is somewhat close to the result discovered in our previous work for a isothermal cure process. ${ }^{16}$ It is clear that the values of activation energy are not a constant, as supposed in many works throughout the whole cure process. At the early stage of cure reaction, the activation energy takes a maximum value, then decreases, and finally remains practically constant.

In general it is apparent that these reactions occurred during curing are very complex and precise kinetics cannot be predicted with the confidence for given compositions and conditions. It has observed that in the basecatalyzed reactions the formation of ether groups is relatively insignificant. ${ }^{17}$ The base catalysis can be further activated by an acid co-catalyst. For example any residual hydroxyl groups can act as internal co-catalyst. Hydroxylic species are present in oligomers of bisphenol A diglycidyl ether resins as part of the structure, and may also be present as impurities.

Poly-carboxylic acid anhydrides can react with epoxy resins to produce ester links, in the presence of acidic or basic catalysts. Tanaka and Kakiuchi proposed the fol-

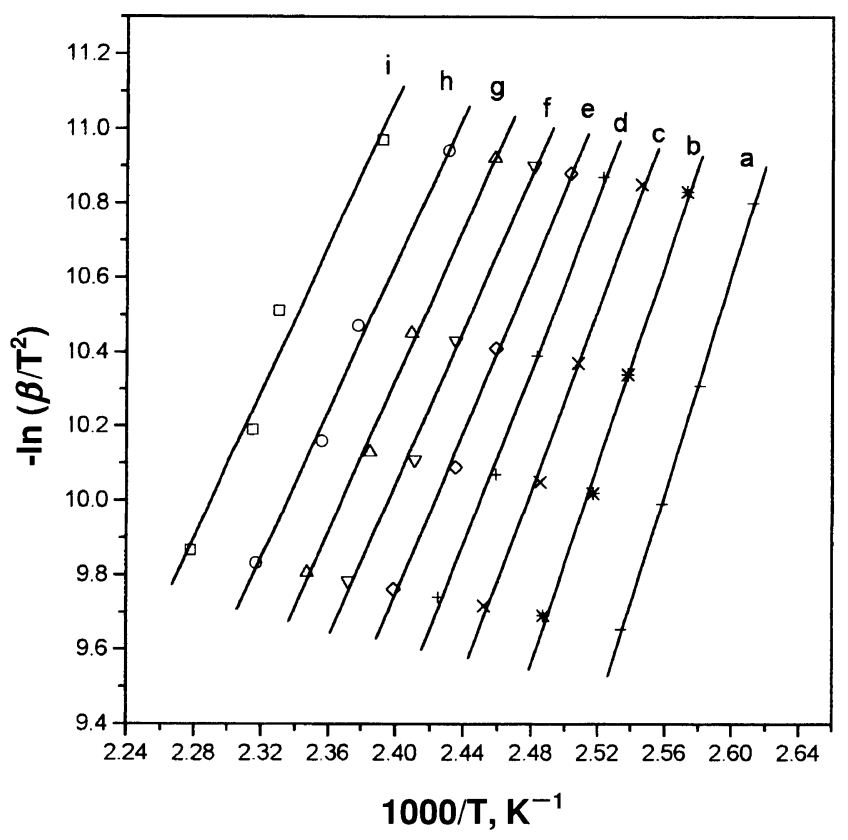

Figure 3. Iso-conversional plots for epoxy molding compounds at different heating rates.

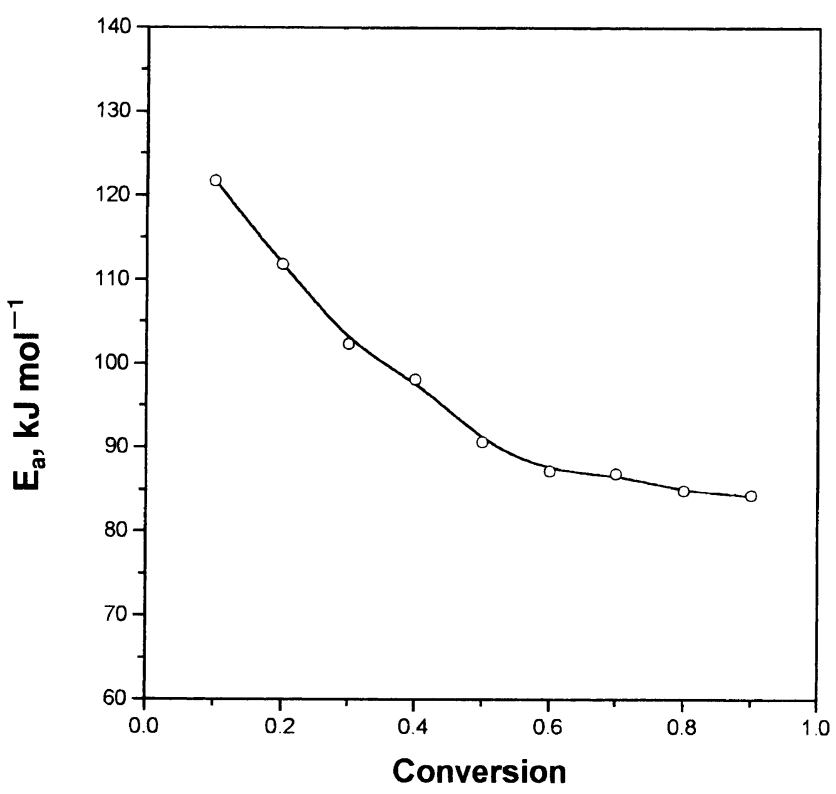

Figure 4. Activation energy of curing as a function of conversion.

lowing scheme for the reaction catalyzed by base (B) with acid co-catalyst (HA): ${ }^{18}$

$B+H A \rightleftharpoons B . H A$

$\mathrm{B} \ldots \mathrm{HA}+\overbrace{\mathrm{CH}_{2}-\mathrm{CH}-}^{\mathrm{a}}=/_{\mathrm{CH}_{2}-\mathrm{CH}-}^{9} \ldots \mathrm{HA} \ldots \mathrm{B}$

(E.H.A.B)

E.H.A.B $+\mathrm{OC}_{-}^{-\mathrm{O}}>\mathrm{CO} \longrightarrow \mathrm{A}-\mathrm{OC}-\mathrm{R}-\mathrm{COOCH}_{2}-\underset{\mathrm{O}}{\mathrm{C}} \mathrm{H}-\mathrm{B}$ 
Table II. Calculation of Arrhenius parameters by ASTM E698-79 method

\begin{tabular}{ccccc}
\hline$\frac{\beta}{\mathrm{K} \mathrm{min}^{-1}}$ & $\frac{T_{p}}{\mathrm{~K}}$ & $\alpha_{p}$ & $\frac{10^{-8} A}{\min ^{-1}}$ & $\frac{E_{a, p}}{\mathrm{~kJ} \mathrm{~mol}^{-1}}$ \\
\hline 3 & 397.5 & 0.438 & 5.07 & \\
5 & 403.8 & 0.427 & 5.83 & 82.2 \\
7 & 410.7 & 0.488 & 5.49 & \\
10 & 416.7 & 0.485 & 5.56 & \\
\hline
\end{tabular}

Table III. The characteristic temperatures of materials by TGA analysis

\begin{tabular}{cccc}
\hline$\frac{T_{\text {Heating rate }}}{\mathrm{K} \mathrm{min}^{-1}}$ & $\frac{T_{\text {onset }}}{{ }^{\circ} \mathrm{C}}$ & $\frac{T_{\max }}{{ }^{\circ} \mathrm{C}}$ & $\frac{\text { Residual }}{\%}$ \\
\hline 5 & 312.3 & 402.5 & 79.04 \\
10 & 339.3 & 412.7 & 78.39 \\
20 & 325.3 & 431.6 & 78.27 \\
50 & 412.6 & 471.7 & 78.29 \\
\hline
\end{tabular}

Propagation can proceed with the repetition of steps (5) to (7) involving the newly formed species, $\mathrm{HA}^{\prime}$, to form a polyester. It was assumed that reaction (7) was rate-controlling.

Therefore, the value of activation energy of $125 \mathrm{~kJ}$ $\mathrm{mol}^{-1}$ at $\alpha \rightarrow 0$ (Figure 4) should be ascribed to the anhydride-epoxide reaction initiated by HA impurities. The value is markedly higher than the results reported by some researchers during the early stage curings $(63$ $\mathrm{kJ} \mathrm{mol}^{-1},<12 \%$ conv. by Peyser, ${ }^{19} 95 \mathrm{~kJ} \mathrm{~mol}^{-1}, 2-22 \%$ conv. by $\mathrm{Mohler}^{20}$ ). However, in all the above cases, the curing process was considered as a single step so that the activation energies obtained were supposed to be constant throughout the entire reaction interval. It does not correspond to the autocatalytic mechanism of the process. As mentioned above, the hydroxyl groups formed during the cure facilitate ring opening. So it is reasonable to expect a decrease (from $\sim 125 \mathrm{~kJ} \mathrm{~mol}^{-1}$ at $\alpha \rightarrow 0$ to about $80 \mathrm{~kJ} \mathrm{~mol}^{-1}$ ) in the activation energy of cure reaction as compared to the very beginning of the process.

The pre-exponential factor can be calculated using the method given in ASTM E698. ${ }^{21}$ It assumes the extent of the reaction at the exotherm peak, $\alpha_{\mathrm{p}}$, is constant and independent of heating rate. Based on the obtained linear relationship between the reciprocal of exotherm peak temperature $\left(T_{\mathrm{p}}\right)$ and the logarithm of the heating rate $\left(\ln \beta / T_{\mathrm{p}}{ }^{2}\right)$, the activation energy can be obtained from the slope of the straight line, and pre-exponential factor be calculated:

$$
A=\frac{\beta_{i} E_{\mathrm{a}, \mathrm{p}}}{R T_{\mathrm{p}, i}^{2}} \exp \left(\frac{E_{\mathrm{a}, \mathrm{p}}}{R T_{\mathrm{p}, i}}\right)
$$

where $T_{\mathrm{p}}$ is the temperature at which maximum conversion rate occurs on DSC curve.

The pre-exponential factor generally reflects the collision frequency of the reactant molecules. During the early stages of the reaction, the monomer concentration is expected to be higher and thus the pre-exponential

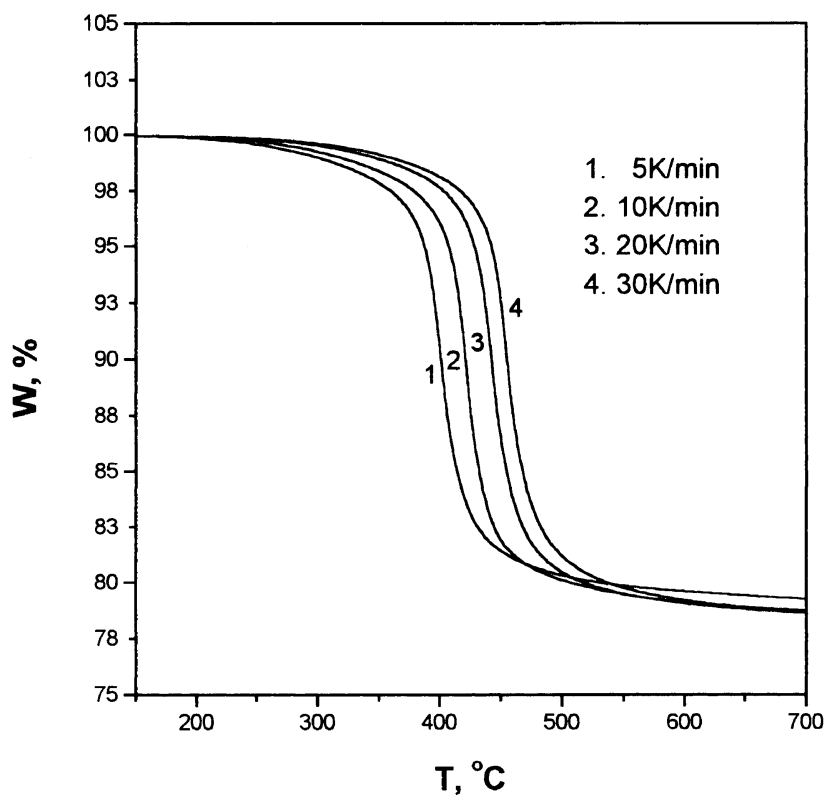

Figure 5. TG thermograms of epoxy molding compounds at various heating rates.

factor is also higher. Higher temperature induces higher motion speed of molecules, and thus increases the possibility of collision and reaction rate. This temperature dependence can be estimated by the Arrhenius equation and expressed with reaction rate constant $k$.

The data from ASTM E698 are given in Table II. As can be seen, the activation energy obtained by this method seems to be little lower than that by the isoconversional analysis. It is not surprising since the conversions at exotherm peak are not a constant as supposed, and ASTM E698 method treats the complex cure process as a single-step reaction of the first order. Compared to the results of iso-conversional analysis, the activation energy evaluated by this method is a single point to the dependence of $E$ on $\alpha$ at $\alpha=\alpha$.

\section{Thermal Degradation}

Figure 5 shows the TG curves of samples at different heating rates from 5 to $30^{\circ} \mathrm{C} \mathrm{min}{ }^{-1}$. The cured epoxy molding compounds show very excellent temperatureresistance although their glass transition temperature $\left(101.3^{\circ} \mathrm{C}\right)$ are much lower than that of aromatic amine cured systems. It may be attributed to the high content of inorganic filler and the high quality resin used in molding compounds.

A number of experimental indices are often used to characterize the process of thermal decomposition, including the onset temperature of decomposition ( $\left.T_{\text {onset }}\right)$, the temperature of the maximum rate of decomposition $\left(T_{\mathrm{d}, \max }\right)$, and the activation energy of decomposition $\left(E_{\mathrm{d}}\right)$.

These indices, especially the characteristic temperatures, are dependent on the rate of heating rate applied during the pyrolysis (listed in Table III). As dynamic DSC scans, the thermograms shifted to higher temperatures as the heating rate increases. The shift of onset to higher temperature with increasing heating rate is due to the shorter time required for a sample to reach a given temperature at the faster heating rates. It is interesting that the TGA curves also significantly shift de- 


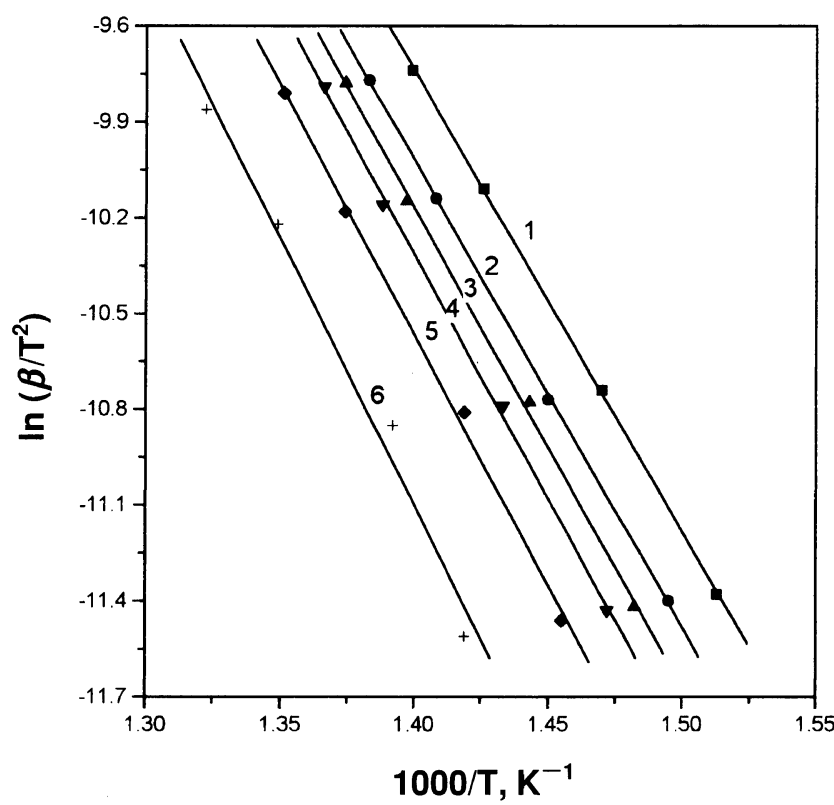

Figure 6. Iso-conversional plots for epoxy molding compounds from different heating rates.

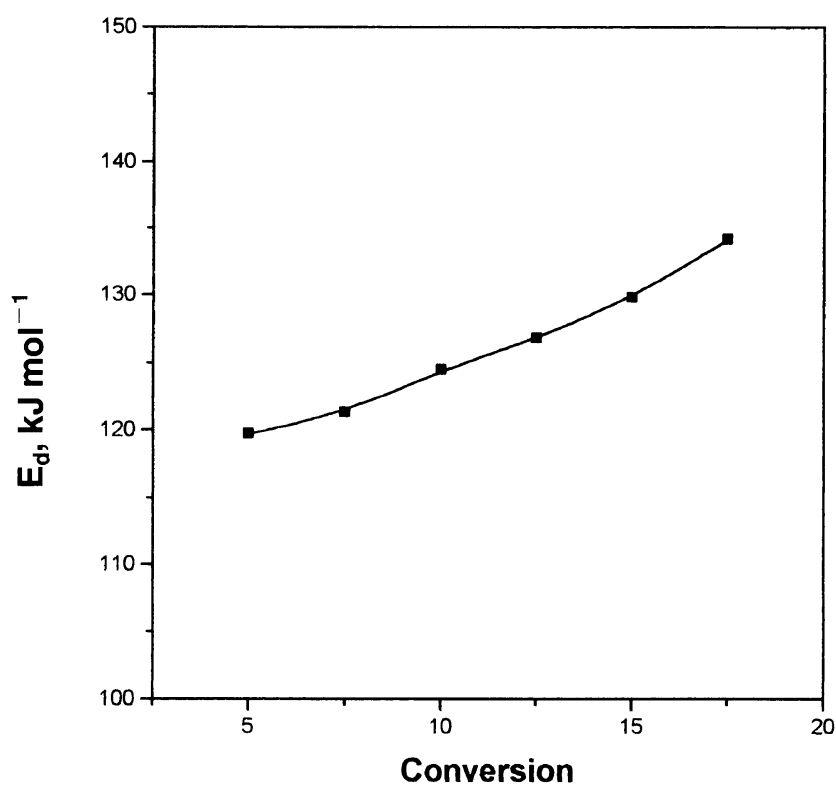

Figure 7. Activation energy of thermal degradation as a function of conversion.

pending on the sample forms and amount. In here, TGA experiments are performed with uniform sample and limited sample weight. Therefore, the curve shift is considered to be practically resulted from the heating rate.

The iso-conversional analysis can been used to characterize the degradation kinetics of thermosets as mentioned above. Figure 6 shows plots of the logarithm of heating rates $v s$. the reciprocal of the analyzed temperature at various conversions resulting in high linearity. The decomposition activation energies at different conversions obtained are plotted in Figure 7. They are in the range from about 120 to $135 \mathrm{~kJ} \mathrm{~mol}^{-1}$. It is worthy to note herein that the activation energy values of cured compounds are not a constant, as supposed in many works throughout the whole degradation process. Unlike the case in curing, it increases with increasing conversion. Detailed mechanisms are still not clear. Perhaps it is related to the fact that the samples do not reach the completed curing under isothermal conditions, and aromatization occurs during degradation.

\section{CONCLUSIONS}

The cure and thermal degradation behavior of epoxy molding compounds can be characterized using an isoconversional model that does not require the knowledge of reaction rate equation $f(\alpha)$. The kinetic results show that the activation energy values for both curing and degradation processes are dependent on the degree of conversion and the cure process is auto-catalytic. The cured materials exhibit very good thermal stability and the activation energy value of decomposition increases with increasing conversion.

Acknowledgement. This work was partly supported by Sang Jin Silicone Co., Ltd. in Korea.

\section{REFERENCES}

1. D. W. Garrett, Proceedings of the A Broader Meaning To Thermosets, SPE-1997, Chicago, IL, p 63.

2. N. Kinjo, M. Ogata, K. Nishi, and A. Kaneda, Adv. Polym. Sci., 88, 1 (1989).

3. S. Matsuoka, S. Quan, H. E. Bair, and D. J. Boyle, Macromolecules, 22, 4093 (1989).

4. X. G. Li, M. R. Huang, and H. Bai, Angew. Makromol. Chem., 256, 9, (1998).

5. E. S. Freeman and B. Carroll, J. Phys. Chem., 62, 394 (1962).

6. T. Osawa, Thermochim. Acta, 100, 109 (1986).

7. A. W. Coats and J. P. Redfern, J. Polym. Sci., Polym. Lett., 3, 917, (1965).

8. C. D. Doyle, J. Appl. Polym. Sci., 6, 639 (1962).

9. M. G. Lu, M. J. Shim, and S. W. Kim, Polym. Eng. Sci., 39, 274 (1999).

10. M. G. Lu, M. J. Shim, and S. W. Kim, J. Appl. Polym. Sci., 75, $1514(2000)$.

11. T. Osawa, Bull. Chem. Soc. Jpn., 38, 1881 (1965).

12. J. H. Flynn and L. A. Wall, J. Res. Nat. Bur. Standards, 70 A, 487 (1966).

13. J. H. Flynn, J. Thermal. Anal., 27, 95 (1983).

14. A. Irabien, C. Santiago, and A. Araiz, J. Therm. Anal., 29, 1131 (1984)

15. S. W. Kim, M. G. Lu, and M. J. Shim, Polym. J., 30, 90 (1998).

16. M. G. Lu, M. J. Shim, and S. W. Kim, J. Appl. Polym. Sci., 71, 2401 (1999).

17. Y. Tanaka and H. Kakiuchi, J. Appl. Polym. Sci., 7, 1063 (1963).

18. Y. Tanaka and H. Kakiuchi, J. Macromol. Sci., Chem., 1, 307 (1966).

19. P. Peyser and W. D. Bascom, J. Appl. Polym. Sci., 21, 2359 (1977).

20. H. Mohler and M. Schwab, Kunststoffe, 71, 245 (1981).

21. Standard Test Method for Arrhenius Kinetic Constants for Thermally Unstable Materials (ANSI/ASTM E698-79), ASTM: Philadelphia, PA, 1979. 